\title{
Integration of Descriptions of Static Structures at Automation of Design Tasks
}

\author{
Olga Novoselova ${ }^{1, *}$ and Anton Sidorov ${ }^{1}$ \\ ${ }^{1}$ MSUT “STANKIN”, 3a, Vadkovsky per., Moscow, 127994, Russia
}

\begin{abstract}
This paper investigates the process of integrating descriptions of static structures in the form of charts and specifications at the stage of conceptual modeling of automated systems, that are developed to solve design tasks. In the process of creating an automated system based on the methodology for automating intellectual labor (MAIL), models of design tasks are formed: initial, conceptual, infological and datalogical. The conceptual model describes the system of concepts of the subject area. The description of conceptual model representation includes specifications and charts: for a static component - F1 and F2, the chart of conceptual structure; for a dynamic component - F3 and F4, the chart depicting a system of subject dependences; F6 and the matrix chart showing the model as a whole including static and dynamic components coordination. The features of constructing diagrams and forming specifications in the formation of a conceptual structure are studied, on the basis of which the method and technique to integrate descriptions of static components for the system of concepts of the subject area are determined. The article is given the integration of descriptions in the form of diagrams and specifications for the static components of conceptual model representations.
\end{abstract}

\section{Introduction}

To date, a number of methods and methodologies for modeling subject tasks and automated systems (AS) have been formed [1, 2, 3, 4]. These methods and methodologies were formed at the stages of development of various approaches to the design of automated systems and programming: structural, object-oriented, cognitive. In this regard, each method or methodology is based on the concept of the approach within which it originated. For structural - functional decomposition, for object-oriented - object decomposition. The cognitive approach is based on a multilevel representation of events and phenomena of the real world, which allows you to simultaneously reflect both a complex multilevel information structure and the structure of various types of processes.

One of the representatives of the cognitive approach is the methodology for the automation of intellectual labor (MAIT) [4, 5]. MAIT was developed in Russia, at the Department of Information Technologies and Computer Systems at MSUT "STANKIN". This methodology is based on the cognitive approach, semiotic approach and its development. MAIT offers an industrial way of creating automated systems and allows you

${ }^{*}$ Corresponding author: ol.novoselova@stankin.ru 
to describe a multi-level information structure and complex formalized algorithms. This type of task includes computational tasks performed in the design of technical objects $[6,7$, 8]. One of the options of the application of methods, including those from MAIT, is the formation of information structures for various applied problems $[9,10]$.

The creation of automated MAIT systems includes a number of modeling stages: initial, conceptual, infological and datalogical [4]. The generated models have a universal representation (conceptual, infological, datalogical) and a representation of subject tasks (initial, conceptual, infological, datalogical). The universal representation is created at three levels of abstraction-abstract, object, and specific; the representation of subject tasks at two levels of abstraction-object and specific.

The initial model representation reflects the process of solving task from the point of view of a subject specialist. The conceptual model view defines the conceptual structure and system of constraints for the task being automated. The infological model representation describes the design of an automated system that is invariant to the program and technical environment and implementers.

Further in the article we will consider the presentation of subject tasks at the stage of conceptual modeling $[4,5,11]$.

The conceptual model view includes the following set of components:

- static (conceptual structure) describes the composition and structure of the static elements of the model;

- dynamic (system of subject dependencies) describes the composition and structure of constraints on the static structure and actually shows the participation of the characteristics of objects (taking into account their location in the static structure) in the actions of algorithms,

- the model as a whole determines the linkage dynamic and static components.

The description for each of the components is formed as special charts and specifications.

When designing an automated system, complex design tasks are decomposed into simpler ones to simplify the process modelling. When forming a model representation for a complex of tasks, models for subtasks are first designed. To obtain a unified description of the system that solves the design problem, the process of integrating model representations of subtasks into the description of the complex of automated system tasks is performed.

\section{Method for integrating static structures of conceptual model representations}

The basic rules for integrating descriptions of model representations are as follows [12, 13]:

1. Integration of subject tasks model representations is carried out separately for models of each level and at each level - on each component (fig. 1).

2. Integration is carried out for each type of the description separately - for charts and specifications.

3. The process of integration includes identifying subtasks merge point, executing integration algorithms and code converting elements of dynamic and static structures.

4. Representation (visualization) of the integration result in the form of charts and specifications for each component of the gained unified model representation.

At a design stage of automated systems, the integration of conceptual model representations on the object level for $n$-subject task is defined as follows [4, 5, 11] (Fig. 1):

$$
K P 2(n)=\bigcup_{i=1}^{m} K P 2_{i}(n)
$$


and includes a number of the sets obtained as a result of integrating sets of the subject categories (2), the static relations (3), the dynamic relations (4) and the relations describing interrelations of static and dynamic components $(5)[14,15,16]$.

$$
\begin{aligned}
& M 2(n)=\bigcup_{i=1}^{m} M 2_{i}(n) \\
& T H 2(n)=\bigcup_{i=1}^{m} T H 2_{i}(n) \\
& \overline{F U 2}(n)=\bigcup_{i=1}^{m} \overline{F U 2_{i}}(n) \\
& R_{2}^{K P}(n)=\bigcup_{i=1}^{m} R_{2}^{K P}{ }_{i}(n)
\end{aligned}
$$
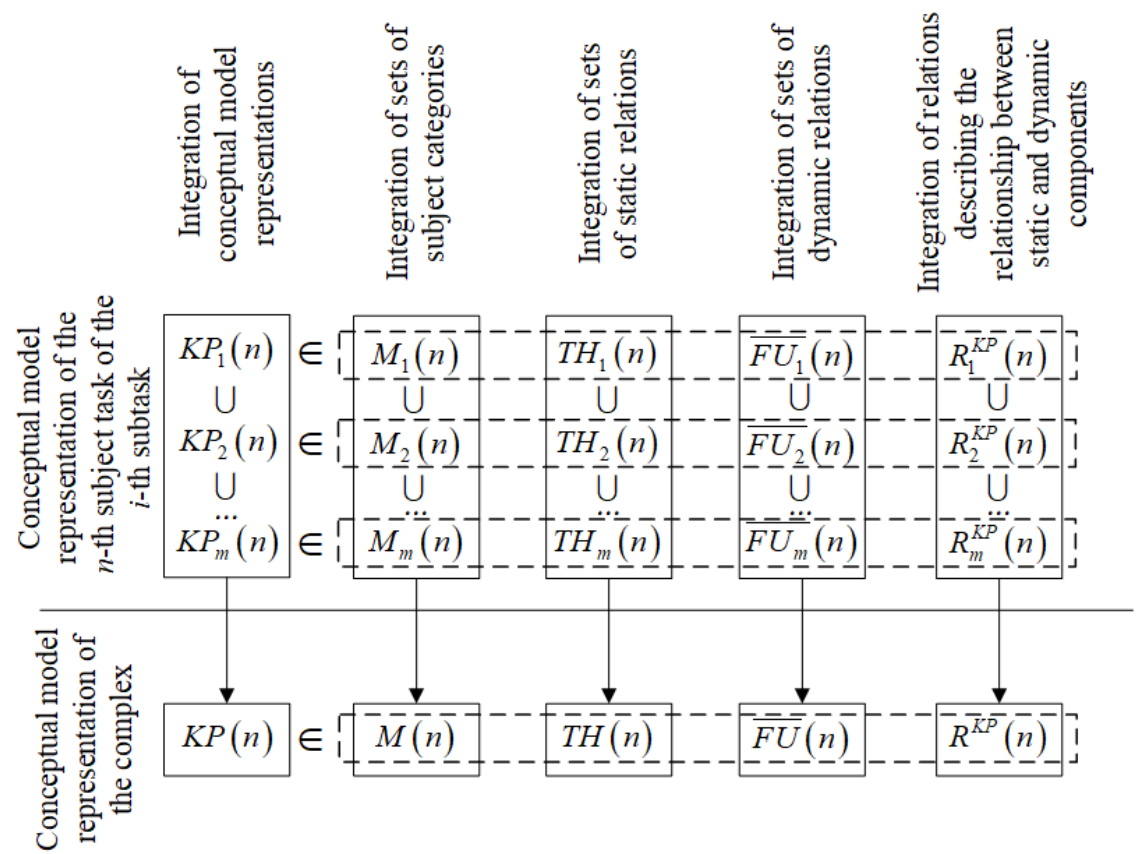

Fig 1. The process of integrating conceptual model representations.

Let's consider the process integrating the static components of conceptual model representation, given in the form of charts and specifications [16].

Descriptions of a complex of subject tasks and each subject task include a conceptual structure - the structure of concepts about the subject area, which consists of many subject categories and many static relations. The conceptual structure is organized as a multi-level structure, which includes the following categories (Fig. 2):

- CYCLE - to represent any product life cycles (S);

- PROCESS - to represent any production processes in the life cycle (P);

- TASK - to represent any production tasks in the process $(Z)$;

- COMPONENT - to represent invariant components of tasks (K);

- OBJECT - to represent various sets of real world objects $(\mathrm{O})$; 
- ATTRIBUTE - to represent any properties of object (R);

- VALUE - to represent elementary categories that fix a specific value of a property of objects (C).

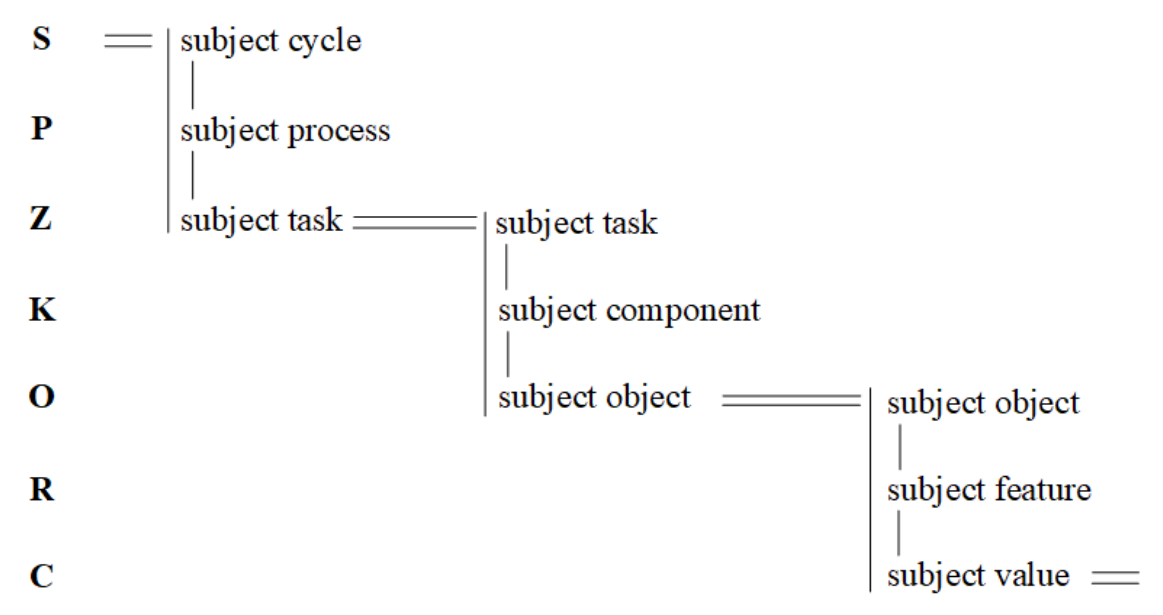

Fig. 2. Conceptual structure at the object level.

The integration of descriptions of conceptual structures in the form of charts is carried out at levels determined by subject categories. The process of integrating conceptual structures begins at the top level "Cycle".

Let's integrate conceptual (static) structure diagrams $(M 2(n), T H 2(n))$, $(M 2(m), T H 2(m))$. At the same time, $(M 2(m), T H 2(m))$ it is a static structure of a subtask, which is integrated into a complex of tasks with a conceptual structure $(M 2(n), T H 2(n))$. As a result of the merging of structures, a new conceptual structure $\left(M 2(n)^{*}, T H 2(n)^{*}\right) \quad$ is obtained, with a single encoding, including $(M 2(n), T H 2(n)),(M 2(m), T H 2(m))$.

Since there is a merging of different diagrams of static structures, we introduce indices $\mathrm{n}, \mathrm{m}$, which determine the belonging of subject categories to the corresponding conceptual structure. It turns out that $a^{n}{ }_{i j}$ is the $j$-th subject category of the $i$-th level of the static structure of the $n$-th object problem, $a^{m}{ }_{k l}$ is the $k$-th object category of the $l$-th level of the static structure of the $m$-th object problem.

At the first step, the initial conceptual structure is determined - the diagram of the $n$-th task or complex and the conceptual structure integrated into it - diagrams of the $m$-th subtask.

Further, the integration process is performed at each level. The integration algorithm is identical at each level. A graphical interpretation of the integration of conceptual structures for the "Component" level is shown in Fig. 3. 


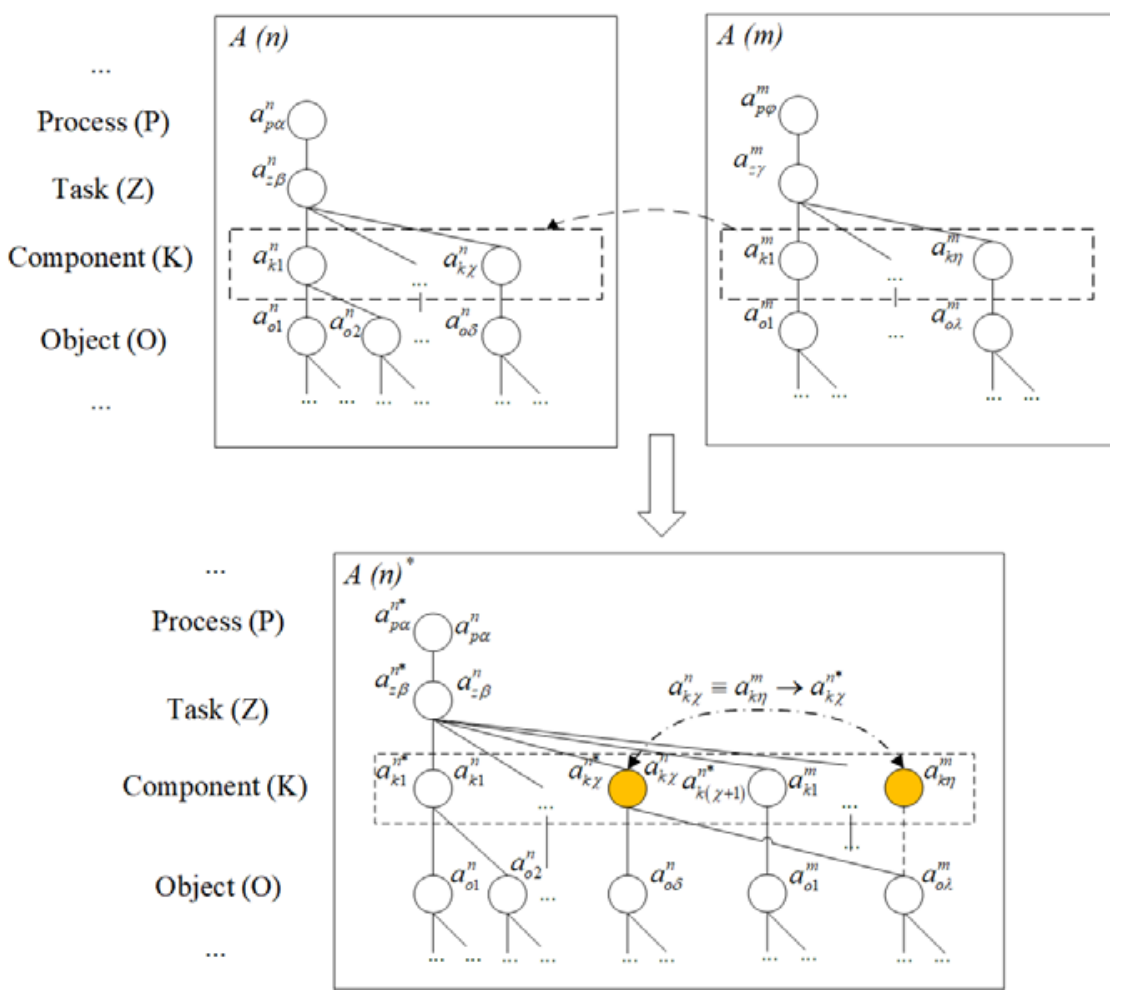

Fig. 3. Graphic interpretation of the integration of subject categories of conceptual structures at the "Component" level.

Formal description of the integration of conceptual structures for the "Component" level:

If $a_{k}^{n}=\left\{a_{k 1}^{n}, \ldots, a_{k \chi}^{n}\right\}, a_{k}^{m}=\left\{a_{k 1}^{m}, \ldots, a_{k \eta}^{m}\right\}$,

then $a_{k}^{n^{*}}=a_{k}^{n} \bigcup a_{k}^{m}$, that is $a_{k 1}^{n} \equiv a_{k 1}^{n^{*}}, \ldots, a_{k \chi}^{n} \equiv a_{k \chi}^{n^{*}}, a_{k 1}^{m} \equiv a_{k(\chi+1)}^{n^{*}}, \ldots, a_{k \eta}^{m} \equiv a_{k(\chi+\eta)}^{n^{*}}$.

When merging at this level, if the categories of the "Component" level are semantically the same (6)

$$
a_{k i}^{n} \equiv a_{k j}^{m}
$$

and

$$
\begin{gathered}
\exists a_{z t}^{n^{*}}: L A_{z}(n)^{*} A_{k}(n)= \\
=\left\{\left(a_{z t}^{n^{*}}, a_{k i}^{n}\right) \mid a_{z t}^{n^{*}} \in A_{z}(n)^{*}, a_{k i}^{n} \in A_{k}(n)\right\}, L A_{z}(n)^{*} A_{k}(m)=\left\{\left(a_{z t}^{n^{*}}, a_{k j}^{m}\right) \mid\right. \\
\left.\mid a_{z t}^{n^{*}} \in A_{z}(n)^{*}, a_{k j}^{m} \in A_{k}(m)\right\},
\end{gathered}
$$

then the final chart will include a category that belongs to the original chart

$$
a_{k i}^{n} \rightarrow a_{k i}^{n^{*}}
$$


otherwise, if $a_{k i}^{n} \not \equiv a_{k j}^{m}$, then both categories fall into the final diagram: $a_{k i}^{n^{*}}, a_{k j}^{n^{*}}$.

After the integration of diagrams at the "Component" level, the code conversion and restoration of links with the level above are carried out.

As a result of the integration process at each level, the following actions are performed: a complete set of subject categories for a complex of tasks is formed, combining subject categories of the original and integrated conceptual structures, recoding of subject categories and restoration of links with subject categories at a higher level $(i-1)$ is carried out.

The description of conceptual model representation includes a set of tables (specifications) $R(n)$. Therefore for the formal description of specifications it is necessary to input indexation [12]:

$$
R(n)=\bigcup_{t} R^{t}(n), R^{t}(n)=\bigcup_{e} R^{t e}(n), R^{t e}(n)=\left\{r_{j}^{t e}\right\}
$$

where $R^{t}(n)$ - subset of the specifications, where $t$ - the type of a component ( $t=1-$ static, 2 - dynamic, 3 - linking the model as a whole), $R^{t e}(n)$ - subset of the specifications, where $t$ - the type of a component $e$ - the number of specification of certain type $(e=1,2, \ldots, \mathrm{m}), r_{j}^{t e}-j$ - tuple of the specification of type $\mathrm{t}$, number e.

Formation of specifications for charts of conceptual structures consists in displaying the obtained encoded static structure in a tabular form (for a task or for a complex of tasks).

The F1 specification fixes the composition of the static structure, the F2 specification allows you to fix the binary connections of the conceptual structure - ordering, composition and arrangement between subject categories both at the same level (ordering) and between different levels (composition). These specifications describe the basic conceptual structure. In fig. 4 provides a description of the F1 specification.

\begin{tabular}{|c|c|c|c|c|c|c|}
\hline \multicolumn{7}{|c|}{ R11(n) (Specification F1) } \\
\hline & $D_{1}{ }^{11}$ & $D_{2}{ }^{11}$ & $D_{3}{ }^{11}$ & $D_{4}{ }^{11}$ & $D_{5}^{11}$ & $D_{6}{ }^{11}$ \\
\hline$r_{1}^{11}$ & Code & $\begin{array}{l}\text { Name of subject } \\
\text { category }\end{array}$ & $\begin{array}{l}\text { Class of } \\
\text { subject } \\
\text { category }\end{array}$ & $\begin{array}{c}\text { Type of } \\
\text { subject } \\
\text { category }\end{array}$ & $\begin{array}{l}\text { Status of } \\
\text { subject } \\
\text { category }\end{array}$ & Note \\
\hline$r_{2}^{11}$ & P1.1 & $\begin{array}{l}\text { the process of } \\
\text { operation the gear } \\
\text { train }\end{array}$ & $\mathrm{P}$ & $\mathrm{T}$ & $\mathrm{P}$ & \\
\hline$r_{3}^{11}$ & $\mathrm{Z} 1.1$ & $\begin{array}{c}\text { the task of operation } \\
\text { the gear train }\end{array}$ & $Z$ & $\mathrm{~T}$ & $\mathrm{P}$ & \\
\hline$r_{4}^{11}$ & $\mathrm{~K} 1.7$ & tool-device & $\mathrm{K}$ & $\mathrm{T}$ & $\mathrm{P}$ & \\
\hline$r 5^{11}$ & O7.1 & node (virtual) & $\mathrm{O}$ & NT & $\mathrm{P}$ & \\
\hline$r_{6}^{11}$ & O7.2 & assembly unit & $\mathrm{O}$ & NT & $\mathrm{P}$ & \\
\hline$r 7^{11}$ & O7.4 & detail & $\mathrm{O}$ & NT & $\mathrm{P}$ & \\
\hline$r 8^{11}$ & R1.1 & node code & $\mathrm{R}$ & $\mathrm{T}$ & $P$ & \\
\hline ... & $\ldots$ & $\ldots$ & $\ldots$ & $\ldots$ & $\cdots$ & $\ldots$ \\
\hline$n_{k}^{11}$ & R3.6 & calculation module & $\mathrm{R}$ & $\mathrm{T}$ & $\mathrm{P}$ & \\
\hline$\cdots$ & $\ldots$ & $\ldots$ & $\cdots$ & $\ldots$ & $\cdots$ & $\ldots$ \\
\hline$r_{f}^{11}$ & R5.1 & center distance & $\mathrm{R}$ & $\mathrm{T}$ & $\mathrm{P}$ & \\
\hline ... & $\ldots$ & $\ldots$ & $\ldots$ & $\ldots$ & $\ldots$ & $\ldots$ \\
\hline
\end{tabular}

Fig. 4. Description of specification F1. 
Thus, it turns out that domains $D_{1}^{11}, D_{2}^{11}, D_{3}^{11}, D_{4}^{11}, D_{5}^{11}, D_{6}^{11}$ contain a description of the composition of the conceptual structure (a list of subject categories).

Using the indexation introduced above for the formal description of specifications, we will describe $R(n)$ - a set of specifications of conceptual model representation for design task $n$ [16]:

$$
R(n)=\left\langle R^{1}(n), R^{2}(n), R^{3}(n)\right\rangle
$$

where $R^{1}(n)$ - subset of descriptions of a static component in the form of specifications (forms F1, F2) (11); $R^{2}(n)$ - subset of descriptions of a dynamic component in the form of specifications (forms F3, F4); $R^{3}(n)$ - subset of descriptions of coordination of model as a whole in the form of specifications (form F6).

$$
R^{1}(n)=\left\langle R^{11}(n), R^{12}(n)\right\rangle
$$

where $R^{11}(n)$ - the description of a static component in the form of the first specification of this type (form F1); $R^{12}(n)$ - the description of a static component in the form of the second specification of this type (form F2).

Integrated specifications represent the combination of two specifications of the $n$-th and $m$-th design tasks, describing the same components by the same kind of forms, where the $m$-th object task is a subtask of the $n$-th task complex:

$$
\begin{aligned}
& R^{11}(n)^{*}=R^{11}(n) \bigcup R^{11}(m), \\
& R^{12}(n)^{*}=R^{12}(n) \bigcup R^{12}(m) .
\end{aligned}
$$

\section{Conclusion}

1. When automating computational design tasks performed in the design of mechanical engineering objects, it is necessary to simulate a multi-level structure of information, a complex formalized process of performing a task, and their mutual coordination.

2. The methodology for the automation of intellectual work is based on a cognitive approach, semiotic approach and its development. MAIT defines an industrial method for creating automated systems, which allows for the stage-by-stage formation of model representations of the subject problem and the future automated system in the form of charts (graphical and matrix) and specifications.

3. The conceptual model representation determines the system of knowledge of the subject area (a system of concepts and constraints for an automated computational task in the design of mechanical engineering objects). It should be noted the importance of the formed conceptual representation, the correctness of which determines in the future the correctness of the interpretation of the descriptions of the automated system project infological and datalogical representations.

4. Integration of graphic descriptions of conceptual structures (charts) allows you to form a single representation of the static component for a complex of subject tasks at the design stage of automated systems, which helps to eliminate redundancy and duplication of elements during the development (expansion) of the system. 


\section{References}

1. Volkova, G.D., Novoselova, O.V., Grigoriev, O.G. Research of methodologies, methods and approaches used in the creation of applied automated systems, Intersectoral information service: scientific and methodological journal, issue 4 (169), pp. 19-31, (2014).

2. Grigoryev, A.V. Database schema method for automatic semantic errors resolving during information systems integration / A. V. Grigoryev, A. A. Kropotin, A. G. Ivashko // 2016 IEEE 10th International Conference on Application of Information and Communication Technologies (AICT). - Azerbaijan, Baku, 2016. - P. 1-7. - DOI: 10.1109/ICAICT.2016.7991683.

3. Garipov, O.A. Conceptual models of heterogeneous data representation / O.A. Garipov, S.V. Zykov // 2010 6th Central and Eastern European Software Engineering Conference (CEE-SECR). - Moscow, Russia, 2010. - P. 50-56. - DOI: 10.1109/CEESECR.2010.5783150

4. G.D. Volkova, Methodology for automating intellectual labor, p. 104, (2013).

5. G.D. Volkova, Theory and practice of automation of intellectual labor, p. 104, (2020).

6. V. Medvedev, A. Volkov, S. Biryukov, Automation of technological preproduction of straight bevel gears, Mechanisms and Machine Science. Volume 81, pp. 133-155, (2020).

7. O.D. Egorov, M.A. Bujnov, Analysis of mechanisms based on their structural and constructive redundancy, Izvestiya Vysshikh Uchebnykh Zavedenii, Seriya Teknologiya Tekstil'noi Promyshlennosti. Volume 2018-January, issue 2, pp. 177181, (2018).

8. V.A. Grechishnikov, R.M. Khusainov, S.Y. Yurasov, R.R. Khaziev, Reliability in gear shaping, Russian Engineering Research. Volume 36, issue 9, pp. 791-795, (2016).

9. Processing Of Conceptual Models Of Subject Problems For Extracting Knowledge From Technical Documentation / N. N. Htwe, G. D. Volkova, K. K. Zan, T. B. Tyurbeeva // 2021 IEEE Conference of Russian Young Researchers in Electrical and Electronic Engineering (ElConRus). - St. Petersburg, Moscow, Russia, 2021. - P. 2185-2189. - DOI: 10.1109/ElConRus51938.2021.9396551.

10. Nikishechkin, P.A. Development of a System for Collect and Process Information on the Operation of Technological Equipment in Digital Production / P. A. Nikishechkin, I. A. Kovalev, A. N. Nikich // 2020 International Multi-Conference on Industrial Engineering and Modern Technologies (FarEastCon). - Vladivostok, Russia, 2020. P. 1-4. - DOI: 10.1109/FarEastCon50210.2020.9271488.

11. G.D. Volkova, Conceptual modeling of design tasks, p. 117, (2015).

12. Novoselova, O. V. Integration of descriptions of conceptual model representations at automation of design tasks / O.V. Novoselova, A.S. Sidorov // IOP Conf. Series: Materials Science and Engineering 709 (2020) 044014.

13. A.S. Sidorov, Integration of descriptions dynamic components of the conceptual model representations, The collection of scientific articles II of the All-Russian scientific conference with the international participation "Information technologies in modeling and management: approaches, methods, decisions", Part 1, pp. 570-576, (2019)

14. A.S. Sidorov, Integration of descriptions dynamic components of the conceptual model representations, The collection of scientific articles II of the All-Russian 
scientific conference with the international participation "Information technologies in modeling and management: approaches, methods, decisions", Part 1, pp. 570-576, (2019).

15. G.V. Eremin, Integration of descriptions of the problem solving process in the form of specifications, The collection of materials I of the All-Russian scientific conference "Information technologies in modeling and management: approaches, methods, decisions", Part 1, pp. 362-368, (2018).

16. A.S. Sidorov, Integration of semantic model representations, The materials of the $\mathrm{X}$ All-Russian scientific-practical conference students, graduate students, educators and industry workers "Information technology and control automation", pp. 294-297, (2019) 